\title{
Qualität im Fokus der Ärzteschaft - die FMH gründet die Schweizerische Akademie für Qualität in der Medizin SAQM
}

Varja A. Meyer, Martina Hersperger
Korrespondenz:

SAQM / Generalsekretariat FMH Elfenstrasse 18

CH-3000 Bern 15

Tel. 0313591111

Fax 0313591112

saqm[at]fmh.ch

\section{Qualität im Berufsalltag - \\ ein Kernanliegen der Ärzteschaft}

Die Qualität der Medizin sichern und fördern - das ist ein Kernanliegen im Alltag von Ärztinnen und Ärzten. Die FMH nimmt dabei eine proaktive und tragende Rolle ein: Sie koordiniert seit mehreren Jahren durch ihre Abteilung Daten, Demographie und Qualität (DDQ) Qualitätsarbeiten innerhalb der Ärzteschaft und lanciert vielfältige Projekte. Im Vordergrund steht dabei stets der Nutzen für Patientinnen und Patienten. Was schon immer in selbstverständlicher Art und Weise Teil ärztlichen Handelns war, soll nun durch Vernetzung und Koordination noch besser gebündelt werden, um Synergien zu nutzen und Doppelspurigkeiten zu vermeiden.

Deshalb hat die FMH gemäss Beschluss der Delegiertenversammlung am 27. November 2012 offiziell die Schweizerische Akademie für Qualität in der Medizin SAQM gegründet. Damit unterstreicht die FMH die Tatsache, dass die Behandlungsqualität, deren Sicherstellung und Erfassung in Ärztehand gehören. Gleichzeitig nimmt sie eine zentrale Rolle in medizinischen Qualitätsfragen ein.

\section{Vielfältige Aufgaben der SAQM}

Die Schweizerische Akademie für Qualität in der Medizin SAQM ist eine ärzteeigene Qualitätsorganisation und zuständig für alle Belange der Qualität in der Medizin. Zu ihren Hauptaufgaben zählt es, den Zusammenhalt innerhalb der Ärzteschaft zum Thema Qualität zu fördern und die Vernetzung von Qualitätsaktivitäten der verschiedenen Ärzteorganisationen zu unterstützen. Als Koordinatorin bei Qualitätsfragen auf nationaler Ebene fördert die SAQM alle Aspekte der ärztlichen Qualitätsarbeit mit Nutzen für Patienten, Angehörige und Ärzte, unterstützt die Entwicklung einer Qualitätskultur und setzt sich für einen hohen Standard von Qualitätsdaten und -projekten ein. Ebenso übernimmt sie die Führung in der Kommunikation zu Qualitätsthemen innerhalb der Ärzteschaft und nach aussen und repräsentiert sie zum Thema Qualität. Künftig wird sich die SAQM auch dem wichtigen Bereich widmen, Qualitätsfragen stärker in der Aus- /Weiter- und Fortbildung der Ärztinnen und Ärzte zu verankern. Dabei geht es nicht um die Qualität der Aus-, Weiter- und Fortbildung selber, sondern um die Integration wichtiger Qualitätsthemen in die bestehenden Curricula.
Für die operative Umsetzung der Arbeiten innerhalb der genannten Tätigkeitsfelder ist nach wie vor die Abteilung DDQ zuständig. So werden auch bereits etablierte Arbeiten sowie laufende Projekte der DDQ in die SAQM integriert und dort weitergeführt.

\section{Abgrenzung zu den einzelnen Ärzteorganisationen}

Für die fachliche Qualität und ihre Umsetzung sind nach wie vor die einzelnen Ärzteorganisationen verantwortlich. Es ist weder das Ziel noch die Aufgabe der SAQM, hier die Hoheit zu übernehmen.

Die SAQM stellt den Nutzen ihrer Aktivitäten für Patienten, Angehörige und Ärzte in den Mittelpunkt. Um Aufgaben, deren Nutzen wissenschaftlich nicht erwiesen ist, kümmert sich die SAQM nur, wenn andere relevante Gründe vorliegen. Als Teil der FMH hat die SAQM keine Legitimation, Qualitätskontrollen durchzuführen, Sanktionen zu ergreifen oder Zertifizierungen zu erteilen - dies ist auch nicht im Sinne der SAQM. Qualitätskontrolle bleibt Sache der Fachgesellschaften im Bereich der Fachfragen und der kantonalen Ärztegesellschaften im Bereich der Umsetzungsfragen. Die SAQM kann grundsätzlich Aufgaben übernehmen, für welche sie jetzt noch nicht zuständig ist; ein solcher Schritt hängt jedoch von einer umfassenden Überprüfung in Bezug auf ihren Sinn und Zweck für die Qualitätsentwicklung ab.

\section{Starker Einbezug der ganzen Ärzteschaft}

Einer der wichtigsten Gründe für die Schaffung der SAQM ist das Anliegen der FMH, ihre Basis noch stärker und direkter in die Ausrichtung ihres Engagements für Qualität in der Medizin einzubinden. Deshalb sind alle medizinischen Fachgesellschaften, kantonalen Ärzteorganisationen sowie die Dachverbände, der Verband Schweizer Assistenz- und Oberärztinnen und -ärzte VSAO und der Verein der Leitenden Spitalärzte der Schweiz VLSS mit je einem Qualitätsdelegierten im neu geschaffenen Forum Qualität vertreten. Das Forum Qualität stellt sicher, dass das fachspezifische und organisationsbezogene Wissen zu Qualitätsfragen in der Medizin in den Arbeiten der SAQM berücksichtigt wird. Es wählt zudem alle vier Jahre aus seinen Mitgliedern vier Delegierte in den Steuerungsausschuss der SAQM.

Im Steuerungsausschuss sitzt nebst den vier Delegierten des Forums Qualität die Ressortleitung $D D Q$ 
(FMH-Zentralvorstand und Abteilungsleitung DDQ). Gemeinsam trägt der Steuerungsausschuss die übergeordnete Verantwortung für alle SAQM-eigenen Projekte und bei externen Projekten die Verantwortung für die Projektbegleitung. Für die Auswahl und Lancierung neuer Projekte dient der Steuerungsausschuss als beratendes Organ für den Zentralvorstand der FMH. Mit diesen Aufgaben und Kompetenzen nimmt der Steuerungsausschuss eine zentrale Rolle in den Strukturen der SAQM ein.

\section{Feedback, Beratung und Dialog}

Bei verschiedensten Arbeiten ist die SAQM auf Knowhow aus Wissenschaft und/oder Praxis angewiesen. Dafür zieht sie vorübergehend oder dauerhaft Experten bei, die eine Beratung sowie ein unabhängiges Feedback gewährleisten.

Daneben strebt die SAQM den Einbezug von Partnern aus dem Schweizer Gesundheitswesen sowie den gegenseitigen Austausch an. Einerseits ermöglicht sie den Partnern Einsitz im Gremium Dialog Qualität, welches der SAQM als Feedbackorgan und beratende Instanz dient. Andererseits ist die SAQM aber auch sehr daran interessiert, in ausgewählten Themen eng mit Partnerorganisationen zusammenzuarbeiten sowie selber in Gremien von Partnerorganisationen vertreten zu sein. Die SAQM versteht sich auch als wichtige Partnerin des geplanten Bundesinstituts für Qualität.

\section{Professionelle Unterstützung punkto Qualitätsfragen}

Mit ihrem weitläufigen Beziehungsnetz ist die SAQM Spezialistin für Vernetzung und Koordination in medizinischen Qualitätsfragen. Sie unterstützt gerne Ärzteorganisationen oder ihre Mitglieder bei ihrer Suche nach Qualitäts-Inputs aus anderen Organisationen und stellt die nötigen Kontakte her. Ausserdem hilft die SAQM bei Fragen zum Thema Qualität

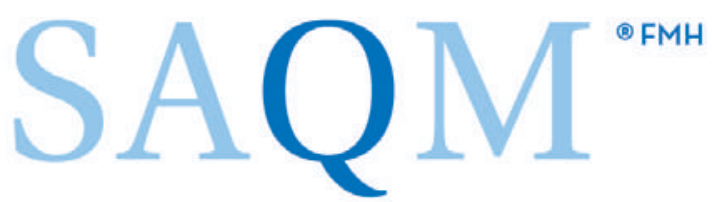

Schweizerische Akademie für Qualität in der Medizin

Am 27. November 2012 hat die FMH die Schweizerische Akademie für Qualität in der Medizin SAQM gegründet. Damit unterstreicht die FMH die Tatsache, dass die Behandlungsqualität, deren Sicherstellung und Erfassung in Ärztehand gehören. Gleichzeitig nimmt sie auf fachlicher Seite eine zentrale Rolle in medizinischen Qualitätsfragen ein.

Weitere Informationen zur SAQM und ihren Tätigkeitsfeldern, Projekten und Dienstleistungen finden Sie unter www.saqm.ch

in der Medizin oder bei Bedarf nach qualitätsbezogenem, fachlichem Support in einem Projekt weiter.

\section{Ausblick aufs kommende Jahr}

Als nächster Schritt werden bis im Frühjahr 2013 das Forum Qualität sowie der Dialog Qualität mit den entsprechenden Delegierten besetzt. Die Wahlen des Steuerungsausschusses finden am ersten Treffen des Forums Qualität am 23. Mai 2013 statt. Jährlich sollen zwei Treffen des Forums Qualität sowie ein Treffen mit den Partnern aus dem Gesundheitswesen im Rahmen des Dialog Qualität stattfinden.

Die SAQM ist bereits vor dem erwähnten ersten Treffen des Forums Qualität aktiv. So sind beispielsweise eine Bedürfnisabklärung sowie eine Inventarisierung der aktuellen Qualitätsarbeiten bei den Ärzteorganisationen in Planung. Beide stellen für die SAQM eine wichtige Basis für ihre zukünftigen Arbeiten dar. 